\title{
Intervención cognitivo-conductual en un caso de "enojo" relacionado con púrpura trombocitopénica inmune
}

\author{
Cognitive-Behavioral Intervention in a Case of "Anger" related to Immune Thrombocytopenic \\ Purpura
}

María L. Escamilla Gutiérrez ${ }^{a}$ y Maetzin Itzel Ordaz Carrillo ${ }^{b}$

\begin{abstract}
:
Immune Thrombocytopenic Purpura (ITP) is a hemorrhagic disease characterized for the destruction of platelets by antibodies. The common treatment is prednisone, this is associated with changes in mood. Because of this, it is a challenge for the psychologist with specialization in Behavioral Medicine to differentiate if it is a psychological problem or not. This case is about a 20 -year-old female patient with ITP. She asked for psychological attention because she had "humor changes" and poor emotional self-control, specifically with anger, since she was taking prednisone. For the evaluation, behavioral interview, clinical observation, STAI, CES-D and Anger's NAS were used, as well as Functional Behavior Analysis based on the Clinical Map of Pathogenesis. The intervention was composed of eleven sessions under Cognitive-Behavioral Therapy, it revealed to have good results and also it was shown that the cause of the problem was psychological due to poor handling of emotions and deficit of certain skills. Anger can be a manifestation of anxiety and depression.
\end{abstract}

\section{Keywords:}

Cognitive-behavioral intervention, anger, depression, generalized anxiety disorder, immune thrombocytopenic purpura

\section{Resumen:}

La Púrpura Trombocitopénica Inmune (PTI) es una enfermedad hemorrágica caracterizada por la destrucción de plaquetas por los anticuerpos. El tratamiento de base es la prednisona, la cual, se asocia con cambios en el estado de ánimo, por eso es un reto para el psicólogo especialista en Medicina Conductual, diferenciarlo de problemas completamente psicológicos. Se trata de una paciente femenina de 20 años con PTI que acude a atención psicológica por "cambios de humor" y deficiente autocontrol emocional, principalmente del enojo, desde que estuvo sometida al tratamiento. Para la evaluación se usó entrevista conductual, observación clínica, IDARE, CES-D y ENA de enojo, así como el Análisis Funcional de la Conducta basado en el Mapa Clínico de Patogénesis. La intervención estuvo compuesta de once sesiones bajo la Terapia Cognitivo Conductual, esta reveló tener buenos resultados y se demostró que la causa del problema era psicológico, por un mal manejo de emociones y déficit de ciertas habilidades. El enojo puede ser una manifestación de ansiedad y depresión.

\section{Palabras Clave:}

Intervención cognitivo-conductual, enojo, depresión, trastorno de ansiedad generalizada, púrpura trombocitopénica inmune

\section{Introducción}

La Púrpura Trombocitopénica Inmunológica (PTI), conocida también como autoinmune, inmune, primaria 0 idiopática, es un trastorno adquirido por una disminución de plaquetas aislada y la ausencia de otras irregularidades que produzcan esta trombocitopenia (menos de 100000 plaquetas $/ \mu \mathrm{l})$ como infecciones, otras enfermedades autoinmunes, consumo de ciertos fármacos, etc. Esta enfermedad hemorrágica se caracteriza por la destrucción prematura de plaquetas a cargo de anticuerpos que están dirigidos contra los antígenos (glucoproteínas) que recubren a las plaquetas y a los megacariocitos. Se encuentran involucrados mecanismos citotóxicos. 1,2

\footnotetext{
a Autor de Correspondencia, Universidad Autónoma del Estado de Hidalgo, Instituto de Ciencias de la Salud, Área Académica de Psicología, https://orcid.org/0000-0002-6318-4519, Email: marilu.escamilla16@ gmail.com

${ }^{\mathrm{b}}$ Universidad Nacional Autónoma de México, Facultad de Estudios Superiores Iztacala, División de Posgrado, https://orcid.org/0000-00028530-7663, Email: psicmaeoc@comunidad.unam.mx
} 
Los síntomas más comunes de PTI son petequias, hematomas y equimosis después de golpes leves, que el paciente por lo regular no recuerda, así como epistaxis, hematuria, melena, menorragia, hematemesis $y$ gingivorragia. En casos más severos, las hemorragias espontaneas aumentan y la probabilidad de sangrado del sistema nervioso central es muy alta. 1

El tratamiento de primera línea es la prednisona, un corticoesteroide que debe ser administrado hasta aumentar el conteo plaquetario. Su uso no puede ser prolongado, debido a los efectos secundarios adversos que desarrolla como: síndrome de Cushing, obesidad, hiperglucemia, hipertensión, acné, osteoporosis, aumento de infecciones, etc. A nivel psicológico puede producir ansiedad, inestabilidad emocional, irritabilidad, depresión, alteraciones de memoria y en el peor de los escenarios, psicosis. 1,3,4

En el caso de los adultos, esta enfermedad tiende a evolucionar hacia la cronicidad (de un 70 a un $80 \%$ de los casos), es decir, la recuperación total es muy rara, aunque en la mayoría de los casos, la enfermedad se presenta de una forma ligera y estable, por lo que difícilmente necesita un tratamiento largo. 2,3

Sin embargo, los pacientes se ven enfrentados a una serie de emociones que dependen del curso de la enfermedad y de la etapa de la vida. Estas emociones pueden estar relacionadas con la enfermedad en sí misma, de la convivencia con ella o como ya se mencionó, del propio tratamiento. En el caso de los adultos, el malestar es resultado del miedo a que se presenten hemorragias incontrolables y consecuencias a largo plazo como la esplenectomía. Los efectos de la PTI tienen un gran impacto en la calidad de vida de los pacientes, pues puede afectar también su productividad laboral. Por lo que es primordial, ofrecer orientación y apoyo psicológico al paciente y su familia. 3

Dado lo anterior, es para el médico conductual un reto determinar si los cambios de humor de los pacientes sometidos a tratamiento con prednisona, son parte de sus reacciones secundarias o es consecuencia de factores psicológicos como el déficit de habilidades y un mal manejo de las emociones.

El objetivo de este artículo es ilustrar la intervención cognitivo-conductual en el caso de una paciente con PTI que refiere "cambios de humor" y un deficiente autocontrol emocional.

\section{Presentación del caso clínico}

Se trata de una paciente femenina de 20 años, soltera, con escolaridad de secundaria, con diagnóstico médico de $\mathrm{PTI}$, presenta como comorbilidad asma actualmente controlada. Al comienzo de la intervención la paciente se encontraba desempleada, sin embargo, poco tiempo después recupera su empleo en un restaurant.

Como motivo de consulta, la paciente señala "Decidí pedir la atención psicológica porque desde que me diagnosticaron la PTI desde hace 7 meses, he estado muy irritable, cambio fácilmente de humor, constantemente estoy enojada, me duele la cabeza y ya no sé cómo controlarme. Al principio, pensé que era por la prednisona, porque la doctora me había dicho que era parte de sus efectos secundarios, pero ya tengo 4 meses que no la tomo y sigo igual" (sic. pac.).

Como antecedentes médicos de importancia refiere padecer asma desde los 7 años, con última crisis a los 12 años. Así como el internamiento y la trasfusión de 3 concentrados eritrocitarios debido a la PTI, siete meses antes de la entrevista psicológica.

Como antecedentes psicológicos patológicos manifiesta no haber recibido atención psicológica o psiquiátrica previa, sin embargo, se describe a sí misma como una persona ansiosa por cualquier detalle y miedosa, desde que era niña.

Desde que le diagnostican asma, su madre se mostró muy sobreprotectora con ella, hasta los 17 años, que sus padres se separan y deja de ser el centro de atención de su madre.

En cuanto a su dinámica familiar, ella vive con su madre con la que frecuentemente discute. Con su padre mantienen una relación cordial. La paciente era el principal sustento económico antes de enfermar de PTI.

\section{Técnicas de evaluación}

Para evaluar se utilizó la entrevista conductual, la observación clínica, el Inventario de Ansiedad RasgoEstado (IDARE),5 la Escala Center for Epidemiological Studies-Depression (CES-D) 6 y ENA de enojo.

\section{Análisis descriptivo de la conducta}

La paciente indica "Desde que me enfermé de PTI, he estado muy irritable, cambio fácilmente de humor, constantemente estoy enojada, me duele la cabeza y ya no sé cómo controlarme, he dejado de ser tolerante con la gente" (sic. pac.).

En cuanto al motivo del enojo menciona "me enojo por muchas cosas, porque no encuentro mi ropa y tengo que buscarla, porque mi mamá me pregunta por mi papá después de que salgo con él, porque está constantemente sobreprotegiéndome, quiere que coma demasiado, me pregunta constantemente si me siento mal, no me gusta que me grite que ya baje a comer, también me enojo cuando mi abuela se queja de que no tiene varias cosas, cuando mi mamá desperdicia el dinero, que me repitan lo que tengo que hacer 
constantemente o cuando alguien me cancela o no llega a las citas" (sic. pac.). Además, ha detectado que después de que se enoja siente mucho calor y dolor de cabeza, por lo que se duerme, el enojo se va, pero despierta sin ganas de hacer nada.

"También me he sentido triste, pues casi ya no salgo ni busco a mis amigos, prefiero quedarme acostada en mi casa. Me siento sola y como si solo viera las cosas negativas de lo que me pasa" (sic. pac.).

"Últimamente, no hago nada durante el día, hace dos meses que dejé mi trabajo en el restaurante por las citas que tenía aquí, sólo he tenido trabajos temporales, así que todo el día me la paso durmiendo, despierto sin ganas y con mucha flojera. Durante la noche no duermo y si lo hago, no descanso [...] por lo regular, me duermo a las 3 de la mañana y despierto alrededor de la 1 de la tarde, pero varía mucho eso, porque a veces me quedo hasta las 6 de la mañana viendo el celular" (sic. pac.).

"Estoy intentando volver a trabajar, ya que cuando trabajo, cambio completamente. Aunque últimamente también me desesperaba la gente ahí, sin embargo, me gusta lo que hago, siempre he tratado con mucha gente" (sic. pac.)

\section{Análisis funcional de la conducta}

El análisis funcional de la conducta se basó en el Mapa Clínico de Patogénesis (ver figura 1).7

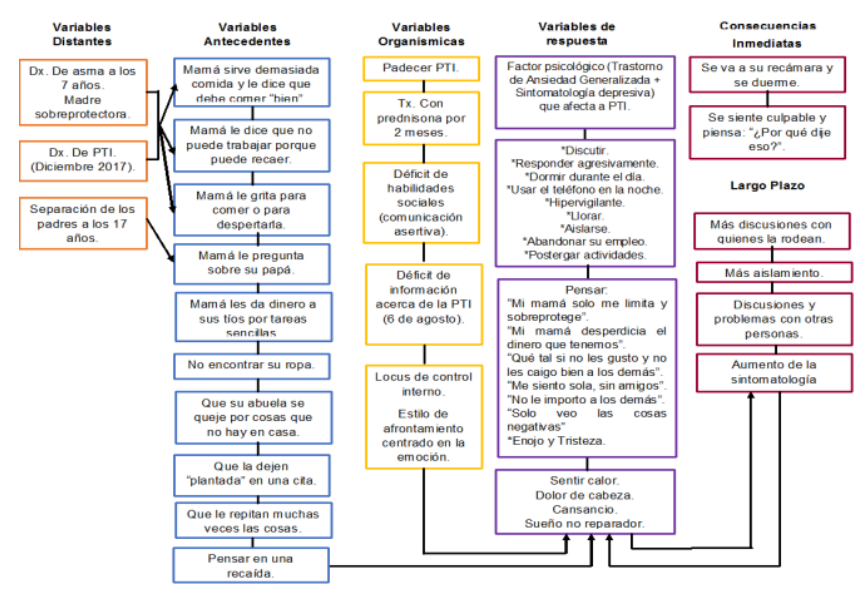

Figura 1. Mapa Clínico de Patogénesis

Se observan variables distantes que parecen estar asociadas etiológicamente con sus síntomas ansiosos y depresivos, tales como a) haber padecido asma desde los 7 años, lo que ocasionó que su madre fuera sobreprotectora con ella, b) el diagnóstico de PTI en el 2017 y la separación de sus padres desde que ella tenía 17 años de edad.

Por otro lado, las variables organísmicas: a) padecer PTI, b) haber estado sometida a tratamiento con prednisona por 2 meses, c) tener un déficit de habilidades sociales, específicamente en la comunicación asertiva, d) Déficit de información de la PTI, detectado hasta el 6 de agosto y e) tener un locus de control interno y utilizar un estilo de afrontamiento centrado en la emoción, son mediadoras de respuesta.

Cuando se presentan ciertas situaciones que operan como estímulos detonantes (es decir, variables antecedentes), como cuando su mamá le sirve demasiada comida y le dice que debe comer bien, cuando le dice que no puede trabajar porque puede recaer, cuando le grita para que vaya a comer o para despertarla, cuando le pregunta por su papá y cuando le da dinero a sus tíos por hacer algunas tareas sencillas. También, en el momento que su abuela comienza a quejarse de cosas que no hay en casa, que le repitan muchas veces las mismas cosas, que la dejen "plantada en una cita" y pensar en una recaída. La paciente manifiesta ciertos comportamientos (variables de respuesta) como: a) discutir, b) responder agresivamente, c) dormir durante el día, d) usar el teléfono durante la noche, e) estar hipervigilante a cualquier molestia, f) llorar, g) aislarse, h) abandonar su empleo e i) postergar sus actividades (dejar para después las citas con medicina conductual), así como pensar: "Mi mamá solo me limita y sobreprotege", "Mi mamá desperdicia el dinero que tenemos", "Que tal si no les gusto y no les caigo bien a los demás", "Me siento sola, sin amigos", "No le importo a los demás", "Solo veo las cosas negativas", se siente enojada y triste. Como reacciones fisiológicas, presenta: a) sensación de calor, b) dolor de cabeza, c) cansancio y d) sueño no reparador. El irse a su recamara y dormirse sin solucionar los problemas, además de sentirse culpable y pensar en "¿por qué dije eso?" (Consecuencias inmediatas) refuerzan las variables de respuesta, lo cual mantiene la sintomatología ansiosa y depresiva. A su vez, esto ha provocado más discusiones con otras personas como con amigos, tíos, etc., que, por tanto, se aislé más, discusiones y problemas con personas con las que antes no tenía problemas, como con las de su trabajo, lo que puede aumentar aún más su sintomatología.

Dado lo anterior, se consideró dirigir el tratamiento a la modificación de las variables de respuesta y a dos de las variables organísmicas (déficit de habilidades sociales y de información de la enfermedad).

\section{Hipótesis Funcional}

Si la paciente recibe un tratamiento cognitivo-conductual compuesto de las técnicas: psicoeducación, respiración pasiva, relajación autógena, control de estímulos, elementos de terapia cognitiva, de solución de problemas y del entrenamiento en habilidades sociales, disminuirá su sintomatología ansiedad y depresión.

\section{Diagnóstico}

A continuación, se presenta el diagnóstico con base en el DSM-5:

Dx. Principal. 300.02 [F41] Trastorno de Ansiedad Generalizada (300.02) + Sintomatología depresiva 
Trombocitopenia Inmune Primaria. Problema de relación con los padres. WHODAS: 22 (Administrado por el entrevistador, 12 preguntas).

\section{Intervención}

Los objetivos de la intervención se pueden observar en el Mapa de Alcance de Metas (Ver figura 2).7

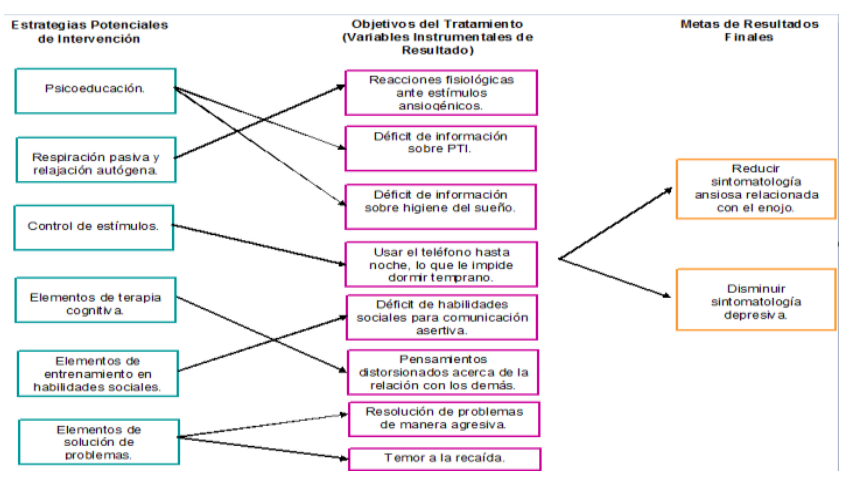

Figura 2. Mapa de Alcance de Metas

El tratamiento estuvo compuesto de 11 sesiones de una hora cada una, desarrolladas en consulta externa durante 6 meses.

Durante las dos primeras sesiones, se evaluó la problemática de la paciente por medio de las técnicas que se mencionaron, se ofreció retroalimentación de los resultados obtenidos, explicándole a la paciente cómo se comportaba por medio del Mapa Clínico de Patogénesis. Posteriormente, se comenzó con la técnica de control de estímulos y psicoeducación de higiene del sueño, para mejorar la calidad del mismo. Se continuó con el entrenamiento en respiración pasiva y relajación autógena para que lograra controlar las reacciones fisiológicas.

Del mismo modo, durante cuatro sesiones se le entrenó en terapia cognitiva de Beck para que identificara la relación entre pensamiento, emociones y conducta. Así como las distorsiones cognitivas que le provocaban enojo y las modificara por pensamientos más adaptativos.

Se le entrenó en comunicación asertiva durante cuatro sesiones, para que pudiera expresar sus emociones y pensamientos de mejor manera. Se le presentaron los "derechos asertivos", la importancia del lenguaje no verbal y la "fórmula de la asertividad", se le modeló con ejemplos cómo ser asertiva, se hicieron ensayos conductuales, se moldeo y se reforzaron los aciertos.

Como parte de las actividades del médico conductual se encuentra acompañar al médico tratante durante la consulta externa, por lo que antes de la sesión número cinco, la paciente acude a su revisión de rutina, y ahí el médico le comenta que sus síntomas son de origen psicológico y ya no un efecto secundario de la prednisona, asimismo, se identifica que la paciente tiene dudas acerca de la PTI que también influyen en la sintomatología mixta, por lo que para esa sesión se brinda psicoeducación de la enfermedad, las medidas de autocuidado, los datos de alarma ante posible recaída y se derribaron mitos.

Durante cuatro sesiones se utilizó la técnica de solución de problemas, con el objetivo de que la paciente aprendiera a solucionar problemas de la vida diaria de forma adaptativa, se hizo especial énfasis en crear un plan de acción ante recaídas de la PTI, situación que también le producía ansiedad.

Finalmente, en la sesión número 11 se realizó la postevaluación, donde la paciente refirió llevar a cabo las técnicas aprendidas.

\section{Resultados}

La intervención cumplió con el objetivo establecido, como se muestra a continuación, la ansiedad, la depresión y el enojo disminuyeron.

En la siguiente figura se puede observar como la percepción de enojo disminuye en la tercera sesión, sin embargo, comienza a aumentar desde la cuarta, se mantiene estable en la cinco, seis y siete, para ir a la baja en sesiones posteriores (Ver figura 3).

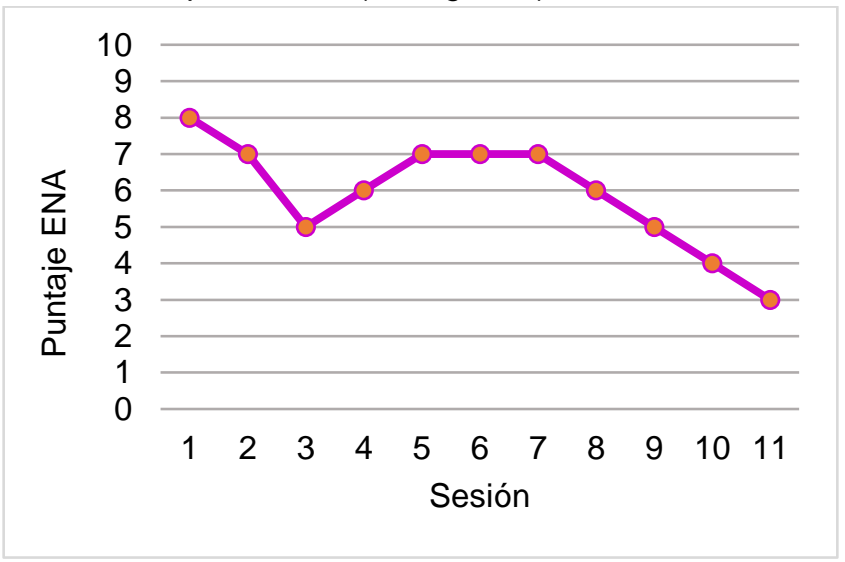

Figura 3. Nivel de enojo percibido a lo largo de la intervención

Los resultados obtenidos por el IDARE y el CES-D son los siguientes (Ver tabla 1).

Tabla 1. Puntajes del IDARE y CES-D antes y después del tratamiento

\begin{tabular}{ccc}
\hline & Antes & Después \\
\hline IDARE (Estado) & 44 (medio) & 29 (bajo) \\
IDARE (Rasgo) & 56 (alto) & 38 (medio) \\
CES-D & 31 (síntomas & 5 (sin síntomas) \\
& depresivos & \\
& significativos) & \\
\hline
\end{tabular}

Los resultados cualitativos también son positivos, ya que la paciente menciona "ya me enojo menos... discuto menos con la gente y hace mucho que no me duele la cabeza" (sic. pac.). "Un día me sentí mal y recordé el plan 
que creamos para venir al hospital, me fue de mucha ayuda" (sic. pac.).

\section{Discusión y Conclusiones}

El objetivo de este caso fue ilustrar como el uso de ciertos medicamentos puede modificar el estado emocional de una persona, y que es labor del médico conductual identificar si ese estado de ánimo es debido al fármaco, en este caso prednisona, o si la génesis del problema es de tipo psicológico, en un mal manejo de emociones y en un déficit de algunas habilidades. Como se pudo observar en este caso particular, la causa es psicológica.

La paciente se describe a sí misma como alguien ansiosa y preocupada por todo (Trastorno de Ansiedad Generalizada), sin embargo, después del diagnóstico de $\mathrm{PTI}$, estos síntomas aumentaron, haciéndose acompañar de sintomatología depresiva. Hay varios estudios que relacionan la ansiedad con síntomas de depresión, pero pocos hablan del enlace entre ansiedad y enojo. El enojo interactúa con síntomas exacerbados de ansiedad. En jóvenes, específicamente, la ansiedad puede expresarse con llanto, irritabilidad y explosiones de ira que a veces puede ser malinterpretado con oposición a padres y maestros.8 Asimismo, el enojo siempre es precedido por ansiedad.9 Dado el papel que el enojo jugaba en relación con los síntomas ansioso-depresivos en el caso de esta paciente y que era la emoción que la paciente identificaba, es que se decide enfocar la intervención en este aspecto, empero, se tiene que tener en cuenta que el enojo es solo un síntoma de la sintomatología mixta. La intervención fue basada en el libro Formulación de casos y diseño de tratamientos cognitivo-conductuales. Un enfoque basado en problemas7 para problemas de ira, obviamente con algunas adecuaciones, con la finalidad de crear un plan de tratamiento más ideográfico. Según los resultados arrojados, el enojo percibido disminuyó hasta las sesiones cuatro y cinco que incrementó, lo cual se puede explicar por los cambios de trabajo que en ese momento se estaban presentando. Los resultados cualitativos demuestran que la paciente detectó cambios positivos desde que se inició el tratamiento.

Derivado de lo anterior, esta intervención puede servir como un parteaguas para problemáticas parecidas a la de esta paciente con PTI.

Para finalizar, entre las limitaciones de este estudio de caso se encuentran: 1) de la evaluación, la necesidad de integrar ENA de ansiedad y depresión, ya que eran las problemáticas principales y el enojo solo era un síntoma, así como, evaluar a profundidad los conocimientos de la enfermedad desde el primer contacto. 2) de la intervención, asegurarse de brindar psicoeducación de la enfermedad al comienzo de la intervención y 3) De los resultados, era necesario, corroborar si existió una generalización a otras situaciones en una sesión de seguimiento.

\section{Referencias}

[1] Jaime JC, Gómez A. Hematología. La sangre y sus enfermedades. 3ra ed. México: McGraw Hill; 2012.

[2] Consejo de Salubridad General. Diagnóstico y tratamiento de púrpura trombocitopénica Inmunológica. Guía de práctica Clínica. México: Gobierno Federal; 2009.

[3]Grupo Europeo de Trasplante Sanguíneo y Médula Ósea. Trombocitopenia Inmune. Una guía práctica para enfermeras y otros profesionales de la salud. España: EBMT; 2011.

[4] Lorenzo P, Moreno A, Lizasoain I, Leza JC, Moro MA, Portpoles A. Velázquez Farmacología Básica y Clínica. 18a ed. Buenos Aires: Médica Panamericana; 2015.

[5] Spielberger CD, Díaz-Guerrero R. Inventario de ansiedad rasgo-estado. México: El Manual Moderno; 1975.

[6] Radloff L. The CES-D Scale: A self-report depression scale for research in the general population. Appl Psychol Meas. 1977; 1:385401 .

[7] Nezu A, Nezu C, Lombardo E. Formulación de casos y diseño de tratamientos cognitivo-conductuales. Un enfoque basado en problemas México: El Manual Moderno; 2006.

[8] Walsh LM, Wolk CB, Becker EM, Jensen M, Beidas RS. The relationship between anger and anxiety symptoms in youth with anxiety disorders J Child Adolesc Psychopharmacol. 2017; 1-17.

[9] Hawkins KA, Cougle JR. Anger problems across the anxiety disorders. Findings from a population- based study. Depress Anxiety. 2011; 28:145-152. 